\title{
Low knowledge and awareness of monoclonal gammopathy of undetermined significance (MGUS) among general practitioners
}

\author{
Charlene M. McShane ${ }^{1 *}$, Blain Murphy ${ }^{1}$, Olinda Santin² and Lesley A. Anderson ${ }^{1}$
}

\begin{abstract}
Introduction: While multiple myeloma (MM) is a rare diagnosis within primary care, its precursor MGUS (monoclonal gammopathy of undetermined significance) is more common, particularly among older populations. Upon first detection, the majority of MGUS patients will be under the care of their General Practitioner (GP)/Family Doctor who is also often the first healthcare professional that patients report symptoms of progression to. However, our previous work with MGUS patients and haematology healthcare professionals has suggested that knowledge and awareness of MGUS is low among GPS.
\end{abstract}

Methods: An online survey was undertaken to investigate knowledge and awareness of MGUS and services needed by GPs/GP trainees to support these patients. The survey was promoted at a large European primary care conference and via social media. Descriptive statistics were utilised to compare participant responses.

Results: In total 58 GPs ( $n=35$ GPs and $n=23$ GP trainees) from 24 countries responded. Overall, self-reported familiarity with the term MGUS was low (mean score: 2.21/5, standard deviation (SD): 1.09), but higher among GPs who reported having at least one MGUS patient (mean score: 2.83/5, SD 0.99). The majority (88.2\%) of GPs/GP trainees stated they would feel uncomfortable discussing MGUS with patients. The increased risk of haematological malignancies was identified by $62.1 \%$ of GPs/GP trainees with MM, lymphoma and myelodysplastic syndromes the most commonly reported cancers associated with MGUS. The majority (81.6\%) of GPs/GP trainees were supportive of patient follow-up via telephone clinics (phlebotomy performed in GP practice with patient management maintained by haematology) but only $27.1 \%$ stated they would be happy to solely manage all low/lowintermediate risk MGUS patients. A laboratory report alerting to the possibility of MGUS or a haematological malignancy was reported as the most useful service which could be implemented to help GPs manage MGUS patients. The need for MGUS focused information and education resources for GPs was also highlighted.

Conclusions: The findings of this study highlight a lack of knowledge and awareness of MGUS among GPs/ GP trainees. The majority of GPs/GP trainees are happy to support haematology in managing these patients but require assistance and support in providing these services.

Keywords: MGUS, Healthcare professionals, Haematology, Myeloma, Communication aids, Family doctors/primary care

\footnotetext{
* Correspondence: c.mcshane@qub.ac.uk

${ }^{1}$ Cancer Epidemiology Research Group, Centre for Public Health, Queen's

University Belfast, Grosvenor Road, Belfast BT12 6BJ, Northern Ireland

Full list of author information is available at the end of the article
}

(c) The Author(s). 2019 Open Access This article is distributed under the terms of the Creative Commons Attribution 4.0 International License (http://creativecommons.org/licenses/by/4.0/), which permits unrestricted use, distribution, and reproduction in any medium, provided you give appropriate credit to the original author(s) and the source, provide a link to the Creative Commons license, and indicate if changes were made. The Creative Commons Public Domain Dedication waiver (http://creativecommons.org/publicdomain/zero/1.0/) applies to the data made available in this article, unless otherwise stated. 


\section{Background}

Multiple myeloma (MM), an incurable B-cell malignancy [1] is the third most common haematological malignancy diagnosed worldwide [2]. MM is proceeded by monoclonal gammopathy of undetermined significance (MGUS) [3, 4], which is estimated to be present in $3.2 \%$ of the population aged 50 years and older [5]. Owing to its asymptomatic nature, MGUS is markedly under diagnosed and is often detected incidentally upon routine blood testing [6, 7]. Clinically, MGUS is defined, by the International Myeloma Working Group, as < $30 \mathrm{~g} / \mathrm{l}$ of serum monoclonal (M) protein, $<10 \%$ plasma cell infiltration in the bone marrow and absence of end organ damage (CRAB criteria - hypercalcaemia, renal insufficiency, anaemia and bone lesions) [8]. The annual rate of progression to MM and related haematological malignancies is between $0.5-1 \%$, and remains elevated beyond 25 years of observation [9, 10]. Follow-up guidelines for MGUS vary internationally, however, most advocate for one annual follow-up visit with relevant myeloma-related investigations $[6,11-$ 13]. In general, it is recommended that these follow-up visits continue indefinitely or until life expectancy becomes limited [6, 11-13].

The majority of patients detected with an M-protein will initially be under the care of their general practitioner (GP)/Primary Care Physician or a clinician outside haematology [11]. Previous research by the study team investigating the psychosocial impact of MGUS among patients has highlighted low awareness and knowledge of MGUS among healthcare professionals outside haematology and in particular, among their GP (Unpublished findings). In response to these findings, the study team undertook a short survey of haematology doctors and nurses attending the Haematology Association of Ireland meeting in October 2016 [14]. Similar findings to the patient study were reported, with haematology healthcare professionals highlighting confusion among patients and GPs alike [14]. Of note, many haematology healthcare professionals reported a combined approach to follow-up involving primary and secondary care is now needed to deal with the increasing number of low/low-intermediate risk MGUS patients being diagnosed [14]. Respondents also recognised that GPs should be supported in this role and provided with guidelines to avoid over-diagnosing and over-referring patients to haematology [14]. Within the current study, we explored GP knowledge and awareness of MGUS and their perceived support needs to manage MGUS patients within primary care.

\section{Methods}

GPs/trainees attending the 22nd WONCA (World Organisation of National Colleges, Academics and Academic Associations of General Practitioners/Family
Physicians) Europe conference in Prague, Czech Republic (http://www.woncaeurope2017.eu/) were invited to participate in an online survey, detailed below. The WONCA Europe Conference is an annual Europe-wide GP/family doctor conference attended by GPs and GP trainees from across the world.

\section{Survey}

In the absence of a validated questionnaire, a short online survey was developed informed by the study team's previous studies with patients and haematology healthcare professionals. The survey was hosted on SurveyMonkey . The survey consisted of 35 questions developed by the study team to capture information relating to awareness and knowledge of MGUS and potential support needs of GPs/GPs in training. The first seven questions were designed to capture respondent demographics and the remaining questions related specifically to MGUS. Familiarity with MGUS was assessed using a likert scale of $1-5$ with 1 equivalent to 'no knowledge/never heard of it' and 5 being 'very familiar'. Multiple choice questions and skip logic were used to reduce the time taken to complete the survey (median time for completion: $9 \mathrm{~min}$ ). All respondents were required to answer the multiple choice/tick box questions but could leave open-ended questions blank. All responses to the survey were anonymous. To increase awareness of the survey, study posters, leaflets and promotion slides were used to advertise the study during the conference. The study team set up a twitter account (@QUB_GPsurvey) and a promotion link was included on the WONCA Europe website. Respondents were provided with the option of being included in a draw for a Samsung Galaxy ${ }^{\oplus}$ tablet.

\section{Data analysis}

The returned survey responses were transferred directly from SurveyMonkey into Microsoft Excel to facilitate data cleaning. The data was analysed to investigate GP awareness and knowledge of MGUS and the support services needed to manage this group of patients. We excluded one respondent from the analysis who stated in the survey that they were not a GP/GP trainee. Descriptive statistics and Chi-squared and Fisher's Exact tests were used to compare participant responses based on variables of interest including GP status (registered GP vs trainee), world region and number of MGUS patients within practice. To assess awareness of MGUS, mean scores and standard deviation were calculated based on a self-reported scale ranging from 1 to 5 with 1 being 'unfamiliar' and 5 being 'very familiar' with MGUS. Missing data was coded as a specific category and excluded from the denominator when calculating responses to questions asked. Responses to open-ended questions were reviewed 
and analysed using content analysis [15]. All tests were two tailed and a $p$-value $<0.05$ was considered statistically significant. All analyses were performed using STATA (version 14, StataCorp, TX, USA).

\section{Ethical approval}

This study received ethical approval from the School of Medicine, Dentistry and Biomedical Sciences Research Ethics Committee, Queen's University Belfast (Ref 17.22).

\section{Results}

\section{Overview of respondents}

In total, $58 \mathrm{GPs} / \mathrm{GP}$ trainees from 24 countries responded to the online survey, Table 1 . Of the 58 respondents, the majority were male (55.2\%), practicing GP's/Family Practitioners $(60.3 \%)$ and had completed their medical degree within the last 5 years (43.1\%). As expected, the majority $(n=47 ; 81 \%)$ of respondents came from European countries (Portugal $n=9$, United Kingdom $n=6$, Netherlands $n=6$, Spain $n=6$, Ireland $n=4$, Greece $n=3$, Croatia $n=3$, Lithuania $n=2$, Luxembourg $\mathrm{n}=2$, Serbia $n=2$, Romania $n=1$, Finland $n=1$, Sweden $n=1$, Switzerland $n=1)$. Six $(10.3 \%)$ respondents came from Asia (Hong Kong $n=1$, Indonesia $n=1$, Israel $n=1$, Lebanon $n=1$, Saudi Arabia $n=1$ and Turkey $n=1$ ) while three (5.2\%) came from The Americas ( $n=1$ USA, $n=1$ Brazil and $n=1$ Mexico) and one from Africa (Tunisia). The majority of respondents worked in metropolitan/ urban areas (72.4\%) and served more than 1000 patients (89.7\%). Just over half of the respondents $(n=30 ; 51.7 \%)$ reported knowing that they had at least one MGUS patient enrolled within their GP practice.

Table 1 GP/GP trainee respondent demographics

\begin{tabular}{|c|c|c|c|c|}
\hline & Total $(n=58)$ & Registered GP $(n=35)$ & GP trainee $(n=23)$ & $p$-value* \\
\hline \multicolumn{5}{|l|}{ Gender } \\
\hline Male & $32(55.2)$ & $22(62.9)$ & $10(43.5)$ & \multirow[t]{2}{*}{0.15} \\
\hline Female & $26(44.8)$ & $13(37.1)$ & $13(56.5)$ & \\
\hline \multicolumn{5}{|l|}{ Continent } \\
\hline Europe & $47(81)$ & $27(77.1)$ & $20(87)$ & \multirow[t]{5}{*}{0.54} \\
\hline The Americas & $3(5.2)$ & $1(2.9)$ & $2(8.7)$ & \\
\hline Asian & $6(10.3)$ & $5(14.3)$ & $1(4.4)$ & \\
\hline Africa & $1(1.7)$ & $1(2.9)$ & 0 & \\
\hline Missing & $1(1.7)$ & $1(2.9)$ & 0 & \\
\hline \multicolumn{5}{|c|}{ Years working as a GP or trainee since completing medical degree } \\
\hline $0-5$ years & $25(43.1)$ & $5(14.3)$ & $20(87)$ & \multirow[t]{4}{*}{$\leq 0.001$} \\
\hline $6-10$ years & $13(22.4)$ & $11(31.4)$ & $2(8.7)$ & \\
\hline $11-20$ years & $13(22.4)$ & $13(37.1)$ & 0 & \\
\hline $20+$ years & $7(12.1)$ & $6(17.1)$ & $1(4.4)$ & \\
\hline \multicolumn{5}{|l|}{ GP Practice } \\
\hline Metropolitan/urban & $42(72.4)$ & $25(71.4)$ & $17(73.9)$ & \multirow[t]{3}{*}{0.52} \\
\hline Rural & $15(25.9)$ & $10(28.6)$ & $5(21.7)$ & \\
\hline Prefer not to say/Not applicable & $1(1.7)$ & 0 & $1(4.4)$ & \\
\hline \multicolumn{5}{|l|}{ Number of patients within GP/Family practice } \\
\hline $0-500$ patients & $3(5.2)$ & $1(2.9)$ & $2(8.7)$ & \multirow[t]{4}{*}{0.83} \\
\hline $501-1000$ patients & $3(5.2)$ & $2(5.7)$ & $1(4.4)$ & \\
\hline $1001-2000$ patients & $25(43.1)$ & $16(45.7)$ & $9(39.1)$ & \\
\hline $2001+$ patients & $27(46.6)$ & $16(45.7)$ & $11(47.8)$ & \\
\hline \multicolumn{5}{|c|}{ Number of MGUS patients that have/are currently within GP/Family practice } \\
\hline None & $8(13.8)$ & $5(14.3)$ & $3(13)$ & \multirow[t]{5}{*}{0.19} \\
\hline 1-10 patients & $24(41.4)$ & $18(51.4)$ & $6(26.1)$ & \\
\hline $11-50$ patients & $5(8.6)$ & $2(5.7)$ & $3(13)$ & \\
\hline 51-100 patients & $1(1.7)$ & $1(2.9)$ & 0 & \\
\hline Don't know/Prefer not to say/Not applicable & $20(34.5)$ & $9(25.7)$ & $11(47.8)$ & \\
\hline
\end{tabular}

*Fisher's exact test used to estimate $p$-value where cell count $<5$ 
Knowledge and awareness of MGUS

When asked to rank familiarity with the term MGUS on a scale of $1-5$ (with 1 being unfamiliar and 5 being very familiar), the respondents reported a mean score of 2.21, standard deviation \pm 1.09 . Registered GPs reported slightly greater familiarity than GP trainees (mean 2.23 vs 2.17) but this difference was not statistically significant. GPs/GP trainees who reported having at least one MGUS patient within their practice reported greater familiarity with MGUS compared to those that did not report any patients or selected 'Prefer not to say' $(2.83 \pm 0.99$ vs $1.54 \pm 0.74 ; p$-value $\leq 0.001)$. The highest mean familiarity score was observed for those GP's/trainees who reported having 11-50 MGUS patients within their practices (3.4 \pm 1.14) although this finding was based on $n=5$ participants. While there were no significant differences between world regions, respondents from Europe reported being more familiar with MGUS [mean score $(2.26 \pm 1.11)$ ] compared to respondents from Asia $(2 \pm 1.26)$, the Americas $(1.67 \pm$ 0.58 ), and Africa ( $n=1$ participant; data not reported to maintain confidentiality).

While just over half (53.5\%) of the respondents recognised MGUS as being a pre-malignant blood disorder associated with production of monoclonal protein, only $25.9 \%(n=15)$ of respondents correctly identified MGUS using the International Myeloma Working Group's definition (serum monoclonal protein $<30 \mathrm{~g} / \mathrm{L}$, clonal plasma cells $<10 \%$ and absence of end organ damage associated with the underlying plasma cell disorder, Fig. 1). GPs/GP trainees with at least one MGUS patient were more likely to answer correctly compared to respondents who reported having no MGUS patients (or prefer not to say) $[n=12(40 \%)$ vs $n=3(10.7 \%) ; p$-value $=0.006]$. GPs/ GP trainees without experience of MGUS patients (or who answered 'prefer not to say) were more likely to report their answer as 'Don't know' compared to respondents with MGUS patients $[n=16(57.1 \%)$ vs $n=6(20 \%)]$.

The majority of participants reported MGUS to be a rare blood disorder ( $n=33 ; 56.9 \%$ ) occurring in those aged 50 years and older ( $n=29 ; 50 \%)$, Fig. 1 . Of the 58 participants, $62.1 \%(n=36)$ correctly identified that MGUS is associated with an increased risk of malignancy. GPs/GP trainees with at least one MGUS patient were more likely to answer correctly $(n=23 ; 76.7 \%)$ compared to respondents who reported having no experience with MGUS patients or prefer not to say $(n=13 ; 46.4 \%$; $p$-value $=0.02)$. The respondents most commonly associated MGUS with progression to MM ( $n=29 / 36$ respondents; $80.6 \%$ ), myelodysplastic syndromes $(n=14 ; 38.9 \%)$ and lymphoma ( $n=12 ; 33.3 \%)$. Of the $40 \mathrm{GPs} /$ trainees who responded to the question on

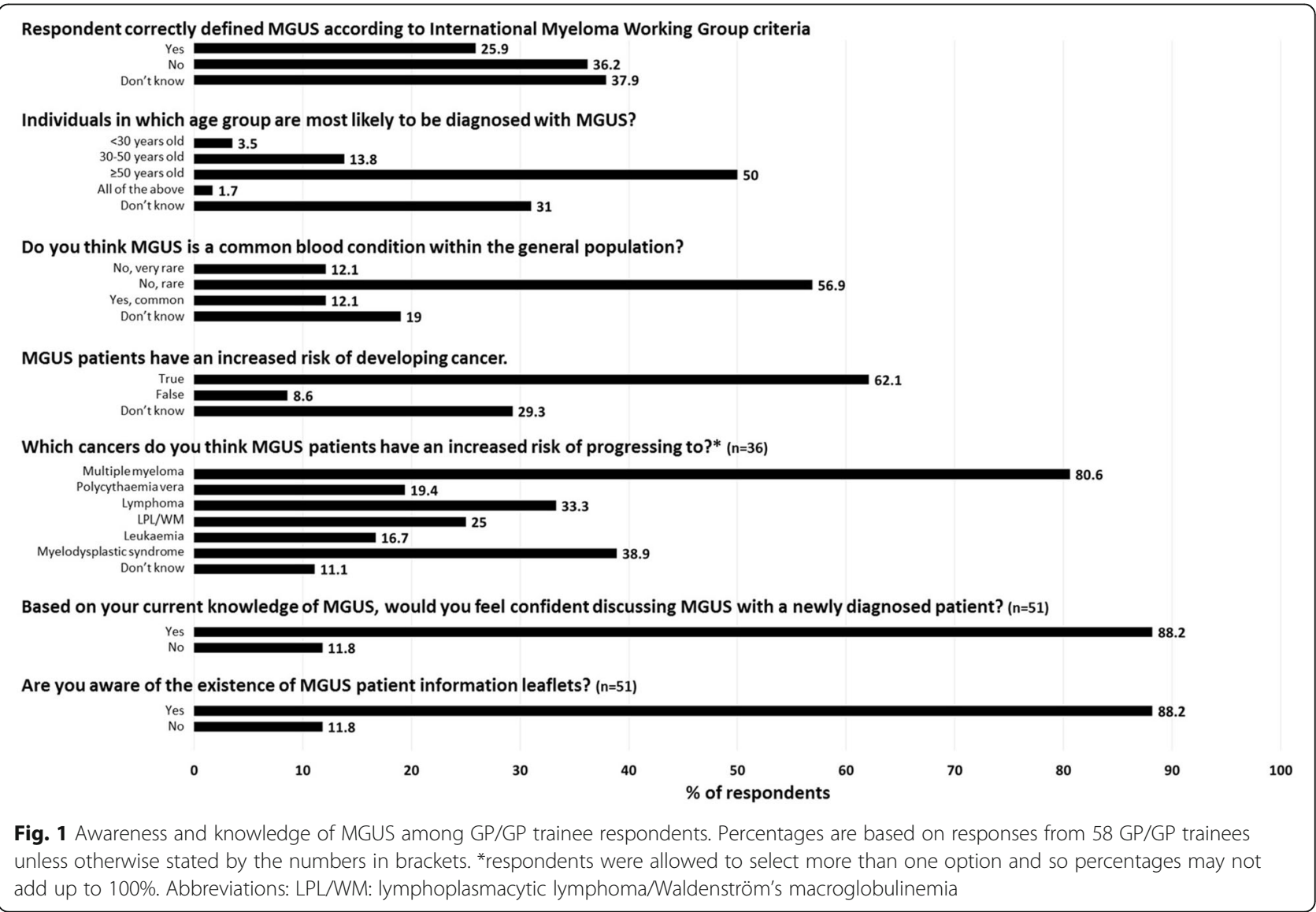


signs and symptoms, 57.5\% $(n=23)$ reported being aware of signs/symptoms associated with progression. Of the 19 respondents who provided additional detail to this question, the most frequent signs/symptoms associated with progression were reported to be bone pain/lesions/osteoporosis ( $n=10 ; 52.6 \%)$, renal disease/abnormalities $(n=7 ; 36.8 \%)$, infections $(n=5 ; 26.3 \%)$, fever $(n=4 ; 21.1 \%)$, anaemia ( $n=3 ; 15.8 \%)$, bleeding $(\mathrm{n}=3 ; 15.8 \%)$, weight loss $(n=3$; $15.8 \%)$, fatigue $(n=2 ; 10.5 \%)$, weakness $(n=2 ; 10.5 \%)$, increase in M protein/worsening of blood tests $(n=2 ; 10.5 \%)$, night sweats $(n=1 ; 5.3 \%)$, and hypercalcaemia $(n=1 ; 5.3 \%)$.

\section{MGUS follow-up}

Of the 51 participants who completed questions on MGUS follow-up, $88.2 \%(n=45)$ stated that based on their current level of knowledge they would not feel comfortable discussing MGUS with a newly diagnosed patient, Fig. 1. This finding did not differ by the number of MGUS patients within the GP practice, however GP trainees were more likely to report feeling uncomfortable $(n=19 ; 100 \%)$ compared to practising GP's $(n=26 ; 81.3 \%$; $p$-value $=0.04)$. Of the 15 respondents who provided additional information, $11(73.3 \%)$ stated that they were uncomfortable due to their limited knowledge/experience of MGUS and would need to update their knowledge before advising patients. While the majority of GPs/GP trainees reported providing information leaflets to some or all of their patients in general (i.e. any medical diagnosis), only $11.8 \%(n=6)$ were aware of the existence of MGUS information leaflets, Fig. 1.
Of the 58 participants, $46(79.3 \%)$ stated that they would either refer all or some patients to haematology if a paraprotein (irrespective of size/isotype) was identified, Fig. 2. Of the 17 respondents who provided additional information, reasons for referral included for diagnostic confirmation and for ruling out malignancy. Some respondents $(n=5)$ stated they would refer patients due to their limited knowledge of MGUS, with one GP stating "Interpreting monoclonal antibodies is more specialised and would be outside a GPs competence to interpret these." While the majority ( $n=21 / 48$ respondents; $43.8 \%)$ deemed haematologists to be the most effective healthcare professionals at following-up MGUS patients, 23\% $(n=11)$ reported a combination approach consisting of haematologists, GPs and nurses as the best strategy. Of the 49 respondents who completed questions regarding follow-up, 40 (81.6\%) were happy for (all or some) MGUS patients to be followed-up via a telephone clinic with a haematology nurse/haematologist while just $27.1 \%$ ( $n=13$ / 48 respondents) stating that they would be happy to follow-up all low/low-intermediate risk MGUS patients within their own GP practice, Fig. 2. Respondents highlighted the need for clear guidelines and guidance from haematology in order to facilitate follow-up. Additional issues highlighted included GP workload and patient safety, for example missing follow up or progression to cancer.

When asked, "What services/information should be provided to GPs/Family Physicians to help them manage MGUS patients?", $53.1 \%$ ( $n=26 / 49$ respondents $)$ of GPs/GP trainees considered laboratory reports alerting

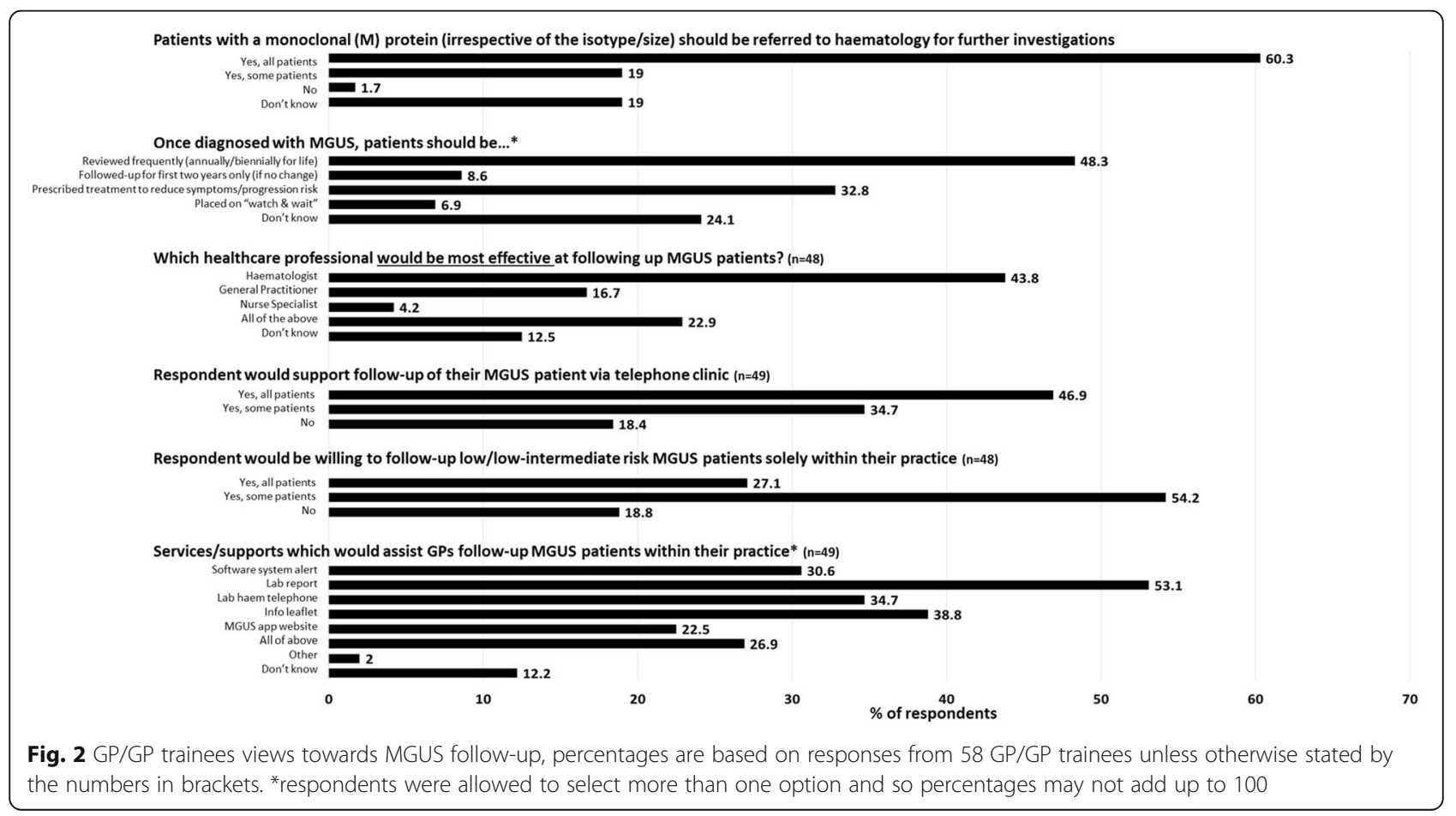


them to the possibility of MGUS or a haematological malignancy as being the most useful service which could be implemented, Fig. 2. Other additional services which ranked highly included, information leaflets $(38.8 \% ; n=$ $19)$, and/or receiving a phone call $(34.7 \% ; n=17)$ from the haematology team at the point of patient diagnosis, and/ or an alert by the clinical software system $(30.6 \% ; n=15)$. Of the 26 respondents who provided additional information, $n=15$ (57.7\%) highlighted the need for information and education resources in the form of information leaflets, journal articles, seminars, webinars, courses and a haematology run website. Respondents were particularly interested in learning more about diagnosis, risk assessment, follow-up, management and signs/symptoms considered red flags. An additional $n=6$ respondents (23.1\%) highlighted their preference for having direct access to a haematology specialist or helpdesk when necessary.

\section{Discussion}

$\mathrm{MM}$ is a rare diagnosis within primary care; the average GP in the United Kingdom (UK) seeing one new MM patient every $8-10$ years [16]. It is perhaps not surprising that our survey revealed a low level of knowledge and awareness of MGUS, the MM precursor among GPs/ trainees. These findings are also in agreement with our previous studies with MGUS patients and haematology healthcare professionals which reported low awareness of MGUS outside of haematology [14]. Despite this, there is increasing government and public demand for primary care to expand its role in cancer prevention, early detection and control, and management within the community [16]. The challenge therefore remains to increase GP awareness and knowledge of MM and its precursor MGUS.

Of the 24 most common cancer sites, MM has previously been associated with the highest percentage of patients visiting their GP three or more times prior to diagnosis [17]. Within the UK, 37\% of MM cases are still diagnosed within emergency care and have been reported to experience poorer outcomes compared to those patients recognised and referred to secondary care by their GPs (one-year survival for GP referral vs emergency presentation: $81 \%$ vs $51 \%$ respectively) [18]. Classified as a 'hard to suspect' cancer [19], MM patients typically present with a myriad of symptoms of a non-specific nature, including, bone pain and extreme fatigue [20]. While just under $60 \%$ of GP's/trainees in this study reported knowing the sign/symptoms associated with MGUS progression, a number of GP's/trainees respondents stated that this area was outside of their expertise and that they would feel uncomfortable discussing the MGUS diagnosis with their patient. The pathway to cancer diagnosis, relies on a relationship of trust between the patient and the doctor. The patient must first be aware of the signs/symptoms to look out for, present to their doctor and their doctor must recognise the possibility of cancer. This relationship is more complex within the UK and similar healthcare systems, where the GP is the gate-keeper to secondary care and specialist treatment. While GPs are not expected to be experts in the field, limited knowledge can lead to delayed identification and negatively impact their patients [17]. Howell and colleagues (2015) previously reported blood cancer patients who did not believe their symptom(s) to be serious were more likely to delay presenting to their doctor [21].

While a 'watch and wait' system is currently recommended for MGUS and smouldering MM patients, recent evidence suggests that initiating treatment early in the MM pathway may have beneficial effects [22]. Furthermore, in a recent Swedish study, MM patients with a prior knowledge of MGUS were reported to have better overall survival compared to patients without a prior MGUS diagnosis [23]. While not possible to establish a causal link between follow-up and survival benefits from this single study, the authors suggest that the findings highlight the importance of clinical follow-up for all MGUS patients, irrespective of risk stratification. However, as a reasonably common phenomenon detected within older populations [5], the number of individuals diagnosed with MGUS is placing an increasing burden on secondary care haematology services. Our recent survey of haematologists in the UK and Irish healthcare systems supported a combined primary and secondary care effort to manage MGUS patient follow-up. The findings from the present study suggests that while this approach would have support from GPs/GP trainees, just over a quarter of respondents were happy to follow-up all low/low-intermediate risk MGUS patients within their GP practice. Within several areas in the UK, outreach haematology monitoring services have been set up for conditions including MGUS and have been reported to improve patient satisfaction and reduce the burden on secondary care [24]. Compliance by general practice for this service has been reported to be very high [24]. This survey highlights an opportunity for haematology specialists to assist GPs' in managing MGUS patients through the provision of clear follow-up guidelines. In addition, laboratory reports (highlighting MGUS or a possible blood malignancy), information leaflets and/or telephone call by haematology team were ranked as the most effective means of communicating with primary care regarding MGUS. Future research including the development of working groups involving primary care and haematology specialists could assist in identifying areas of misunderstanding and in developing relevant resources.

This study is the first to assess knowledge and awareness of MGUS among GPs/primary care physicians. While the generalisability of our findings may be limited by the sample size, we report findings from general 
practitioners/trainees working in 24 countries and across 4 continents'. Furthermore, it is well recognised that GPs are a difficult group to research owing to their work time constraints. The online nature of the survey and restriction to English speakers may have further impacted the generalisability of the findings however, the survey was promoted at a European general practitioner conference and via social media. Similar responses were reported by all respondents suggesting that the findings are representative and could be applied to other regions and healthcare services.

\section{Conclusions}

In conclusion, the findings of this study highlight a lack of knowledge and awareness of MGUS among general practitioners/trainees. These findings are important as the number of MGUS patients detected continues to increase and follow-up potentially transitions towards primary care. Our survey suggests the majority of GPs/trainees are happy with this transition but require assistance and support from haematology in providing these services.

\section{Abbreviations}

GP: General Practitioner; MGUS: Monoclonal gammopathy of undetermined signficiance; MM: Multiple myeloma; SD: Standard deviation; UK: United Kingdom; WONCA: World Organisation of National Colleges, Academics and Academic Associations of General Practitioners/Family Physicians

\section{Acknowledgements}

The authors would like to take this opportunity to thank the organisers of the WONCA Europe 2017 conference and the General Practitioners/Trainee's for participating in this study.

\section{Funding}

At the time of writing, Dr Charlene McShane was in receipt of a Cancer Research UK Population Science Postdoctoral Research Fellowship (C51094) A18267)

\section{Availability of data and materials}

The dataset which was generated and analysed for this study is not publicly available but could be made available from the corresponding author on reasonable request.

\section{Authors' contributions}

The idea and design for this project was developed and led by CMcS and LAA. CMCS analysed the data. CMCS wrote the first draft of the manuscript. All authors (CMCS, BM, OS and LAA) assisted in the interpretation of the data, and reviewed and provided feedback on the final version of the manuscript. All authors read and approved the final manuscript.

\section{Ethics approval and consent to participate}

This study received ethical approval from the School of Medicine, Dentistry and Biomedical Sciences Research Ethics Committee, Queen's University Belfast (Ref 17.22). Participants were informed that participation was voluntary, that all responses were anonymous and that the study would be published in a peer-reviewed journal. Participation in the survey implied consent.

\section{Consent for publication}

Not applicable.

\section{Competing interests}

The authors declare that they have no competing interests.

\section{Publisher's Note}

Springer Nature remains neutral with regard to jurisdictional claims in published maps and institutional affiliations.

\section{Author details}

${ }^{1}$ Cancer Epidemiology Research Group, Centre for Public Health, Queen's University Belfast, Grosvenor Road, Belfast BT12 6BJ, Northern Ireland. ${ }^{2}$ School of Nursing and Midwifery, Queen's University Belfast, Belfast, Northern Ireland.

Received: 9 February 2018 Accepted: 10 April 2019

Published online: 14 May 2019

References

1. Ravi P, Kumar SK, Cerhan JR, Maurer MJ, Dingli D, Ansell SM, et al. Defining cure in multiple myeloma: a comparative study of outcomes of young individuals with myeloma and curable hematologic malignancies. Blood Cancer J. 2018:8:26.

2. Bray F, Ferlay J, Soerjomataram I, Siegel RL, Torre LA, Jemal A. Global cancer statistics 2018: GLOBOCAN estimates of incidence and mortality worldwide for 36 cancers in 185 countries. CA Cancer J Clin. 2018;68(6):394-424.

3. Landgren $\mathrm{O}$, Weiss BM. Patterns of monoclonal gammopathy of undetermined significance and multiple myeloma in various ethnic/ racial groups: support for genetic factors in pathogenesis. Leukemia. 2009;23:1691-7

4. Weiss BM, Abadie J, Verma P, Howard RS, Kuehl WM. A monoclonal gammopathy precedes multiple myeloma in most patients. Blood. 2009;113: 5418-22.

5. Wadhera RK, Rajkumar SV. Prevalence of monoclonal gammopathy of undetermined significance: a systematic review. Mayo Clin Proc. 2010; 85:933-42.

6. Kyle RA, Durie BGM, Rajkumar SV, Landgren O, Blade J, Merlini G, et al. Monoclonal gammopathy of undetermined significance (MGUS) and smoldering (asymptomatic) multiple myeloma: IMWG consensus perspectives risk factors for progression and guidelines for monitoring and management. Leukemia. 2010;24:1121-7.

7. Therneau TM, Kyle RA, Melton LJ, Larson DR, Benson JT, Colby CL, et al. Incidence of monoclonal gammopathy of undetermined significance and estimation of duration before first clinical recognition. Mayo Clin Proc. 2012; 87:1071-9.

8. The International Myeloma Working Group. Criteria for the classification of monoclonal gammopathies, multiple myeloma and related disorders: a report of the international myeloma working group. Br J Haematol. 2003; 121:749-57.

9. Kyle RA, Therneau TM, Rajkumar SV, Offord JR, Larson DR, Plevak MF, et al. A long-term study of prognosis in monoclonal gammopathy of undetermined significance. N Engl J Med. 2002;346:564-9.

10. Turesson I, Kovalchik SA, Pfeiffer RM, Kristinsson SY, Goldin LR, Drayson MT, et al. Monoclonal gammopathy of undetermined significance and risk of lymphoid and myeloid malignancies: 728 cases followed up to 30 years in Sweden. Blood. 2014;123:338-45.

11. Bird J, Behrens J, Westin J, Turesson I, Drayson M, Beetham R, et al. UK myeloma forum (UKMF) and Nordic myeloma study group (NMSG): guidelines for the investigation of newly detected M-proteins and the management of monoclonal gammopathy of undetermined significance (MGUS). Br J Haematol. 2009;147:22-42.

12. Berenson JR, Anderson KC, Audell RA, Boccia RV, Coleman M, Dimopoulos MA, et al. Monoclonal gammopathy of undetermined significance: a consensus statement. Br J Haematol. 2010;150:28-38.

13. van de Donk NWCJ, Palumbo A, Johnsen HE, Engelhardt M, Gay F, Gregersen $\mathrm{H}$, et al. The clinical relevance and management of monoclonal gammopathy of undetermined significance and related disorders: recommendations from the European myeloma network. Haematologica. 2014;99:984-96

14. McShane CM, Murphy B, Lim KH, Anderson LA. Monoclonal gammopathy of undetermined significance as viewed by haematology healthcare professionals. Eur J Haematol. 2018;100:20-6.

15. O'Cathain A, Thomas KJ. \&quot;Any other comments?\&quot; Open questions on questionnaires - a bane or a bonus to research? BMC Med Res Methodol 2004;4:25. 
16. Rubin FRCGPG, Hunter D, Earle C, Grunfeld E, Lyratzopoulos HG, Wardle J, et al. The lancet oncology commission the expanding role of primary care in cancer control. Lancet Oncol. 2015;16:1231-72.

17. Lyratzopoulos G, Neal RD, Barbiere JM, Rubin GP. Abel G a. variation in number of general practitioner consultations before hospital referral for cancer: findings from the 2010 National Cancer Patient Experience Survey in England. Lancet Oncol. 2012;13:353-65.

18. Elliss-Brookes L, McPhail S, Ives A, Greenslade M, Shelton J, Hiom S, et al. Routes to diagnosis for cancer - determining the patient journey using multiple routine data sets. Br J Cancer. 2012;107:1220-6.

19. Lyratzopoulos G, Wardle J, Rubin G. Rethinking diagnostic delay in cancer: how difficult is the diagnosis? BMJ (Clin Res Ed). 2014;349:97400.

20. Shephard E, Neal R, Rose P, Walter F, Litt E, Hamilton W. Quantifying the risk of multiple myeloma from symptoms reported in primary care patients: a large case-control study using electronic records. Br J Gen Pract. 2015;65:e106-13.

21. Howell DA, Warburton F, Ramirez A-J, Roman E, Smith AG, Forbes LJL. Risk factors and time to symptomatic presentation in leukaemia, lymphoma and myeloma. Br J Cancer. 2015;113:1114-20.

22. Mateos M-V, Hernández M-T, Giraldo P, de la Rubia J, de Arriba F, Corral LL, et al. Lenalidomide plus dexamethasone for high-risk smoldering multiple myeloma. N Engl J Med. 2013;369:438-47.

23. Sigurdardottir EE, Turesson I, Lund SH, Lindqvist EK, Mailankody S, Korde N, et al. The role of diagnosis and clinical follow-up of monoclonal Gammopathy of undetermined significance on survival in multiple myeloma. JAMA oncology. 2015;1:168-74.

24. Rawstron AC, Jones RA, Ferguson C, Hughes G, Selby P, Reid C, et al. Outreach monitoring service for patients with indolent B-cell and plasma cell disorders: a UK experience. Br J Haematol. 2007;139:845-8.

Ready to submit your research? Choose BMC and benefit from:

- fast, convenient online submission

- thorough peer review by experienced researchers in your field

- rapid publication on acceptance

- support for research data, including large and complex data types

- gold Open Access which fosters wider collaboration and increased citations

- maximum visibility for your research: over $100 \mathrm{M}$ website views per year

At $\mathrm{BMC}$, research is always in progress.

Learn more biomedcentral.com/submissions 